\title{
Post-Ablation Cavity Evaluation: A Prospective Multicenter Observational Clinical Study to Evaluate Hysteroscopic Access to the Uterine Cavity 4 Years after Water Vapor Endometrial Ablation for the Treatment of Heavy Menstrual Bleeding
}

\author{
D. Alan Johns, MD, Jose G. Garza-Leal, MD, Michael P. Diamond, MD, and Micah Harris, MD \\ From the Baylor Scott \& White Research Institute (Dr. Johns), Fort Worth, Texas, Hospital Universitario Dr. José E. González, Universidad Autónoma de \\ Nuevo León (Dr. Garza-Leal), Monterrey, Nuevo León, Mexico, and AEGEA Medical (Drs. Johns, Diamond, and Harris), Menlo Park, California.
}

ABSTRACT Study Objective: Patients who have undergone endometrial ablation may present a diagnostic challenge when they subsequently develop vaginal bleeding, pelvic pain, or postmenopausal bleeding. Extensive scarring of the uterine cavity often precludes evaluation and/or conservative treatment. For further research on this topic, we performed hysteroscopic examination in study subjects a mean duration of 4 years after they had undergone water vapor endometrial ablation.

Design: Prospective, multicenter, observational clinical study.

Setting: Eight private practice or outpatient sites in the United States and Mexico.

Patients: Seventy subjects who had completed their 36-month follow-up in the AEGEA Pivotal Trial.

Interventions: Diagnostic hysteroscopy.

Measurements and Main Results: The subjects were screened for general health and infection and underwent diagnostic hysteroscopy. Menstrual bleeding status was recorded. The video of the hysteroscopic examination was analyzed by an independent reviewer, who assessed uterine cavity access and visualization of the cornua and tubal ostia as well as characterized adhesions on the basis of the criteria by March et al. An independent reviewer also subjectively assessed whether Pipelle endometrial biopsy or intrauterine device placement would be feasible. Uterine cavity access was achieved in $90 \%$ (63/70) of subjects. Among subjects with cavity access, the cornua and ostia were visualized in 79\% (50/63) and adhesions were absent in $75 \%$ (47/63), with only 2 women having severe adhesions (3\%, 2/63). Biopsy was projected to be feasible in $86 \%(62 / 70)$ and intrauterine device placement in 60\% (42/70) of all subjects. The subjects' bleeding statuses were not correlated with uterine cavity access. The results were consistent for subjects with large uterine cavities and International Federation of Gynecologic and Obstetrics type II to VI myomas $\leq 4 \mathrm{~cm}$.

Conclusion: Water vapor endometrial ablation preserved an accessible uterine cavity and visualization of the ostia in most subjects, with minimal incidence of severe adhesions, a mean of 4 years after the ablation procedure. Journal of Minimally Invasive Gynecology (2020) 27, 1273-1280. @ 2019 AAGL. This is an open access article under the CC BY-NC-ND license. (http://creativecommons.org/licenses/by-nc-nd/4.0/)

Keywords: $\quad$ Ablation; Adhesions; Hysteroscopy; Menorrhagia; Vapor

This study was sponsored by AEGEA Medical, Menlo Park, California. The authors received research-related support for the conduct of this study. Dr. Johns and Dr. Diamond are consultants to AEGEA Medical, and Dr. Harris is the medical director of AEGEA Medical.

Portions of this manuscript have been accepted for oral presentation at the AAGL Global Conference, Vancouver, British Columbia, November 11, 2019.

Corresponding author: Micah Harris, MD, 721 W Luke Ave, Phoenix AZ 85013 .

E-mail: micahharris697@msn.com

Submitted June 25, 2019, Revised September 9, 2019, Accepted for publication September 11, 2019.

Available at www.sciencedirect.com and www.jmig.org
Endometrial ablation is a minimally invasive treatment for heavy menstrual bleeding due to benign causes (primarily, abnormal uterine bleeding-endometrial, AUB-E) in women who have completed childbearing. With the increase in the use of endometrial ablation, potential issues associated with it have become commonplace, with issues often manifesting many years after the original treatment. Recurrent heavy bleeding, cyclic pelvic pain with or without bleeding, and postmenopausal bleeding may occur and require uterine cavity evaluation, ideally using hysteroscopy or endometrial biopsy.

The treatment of patients presenting with these symptoms can be challenging for a gynecologist and may lead to 
situations in which the patients are subjected to hysterectomy because the uterine cavity can be neither accessed nor evaluated. A lack of uterine cavity access because of the formation of adhesions after ablation has been observed and documented as a long-term sequela of endometrial ablation [1]. If long-term access to the uterine cavity after endometrial ablation were possible, less invasive diagnostic or therapeutic interventions could be offered.

Water vapor ablation (AEGEA Medical, Menlo Park, CA) is a U.S. Food and Drug Administration-approved endometrial ablation technique that uses rapid energy transfer of water vapor as it changes from the vapor state to liquid droplets. Water vapor fills the uterine cavity, regardless of its shape or configuration, and transfers energy onto the exterior of the cell membranes. With condensation from gas to liquid, energy is released, which in turn produces the desired tissue effect [2]. Post-treatment histologic studies of the endometrium and myometrium in subjects who had undergone water vapor ablation revealed no carbonization or heat fixation of tissue $[3,4]$. Furthermore, the conforming nature of water vapor allowed the inclusion of this technique in the pivotal clinical trial of subjects with uterine cavity lengths of up to $12 \mathrm{~cm}$ and nonobstructing International Federation of Gynecologic and Obstetrics (FIGO) type II to VI leiomyomata $\leq 4 \mathrm{~cm}$ [2]. Subjects with uterine septa less than one-third the cavity length and Essure contraceptive tubal inserts (Bayer Corporation, Whippany, NJ) were not excluded from the pivotal trial. This Post-Ablation Cavity Evaluation (PACE) study was designed to evaluate long-term hysteroscopic uterine cavity access after water vapor ablation in a cohort comprising subjects who had completed 36 months of follow-up in the pivotal trial.

\section{Materials and Methods}

A prospective, multicenter, single-arm, observational study to evaluate postablation uterine cavity access was conducted at 8 study centers in the United States and Mexico from September 2018 to March 2019. The first study cohort was evaluated at 7 sites in the United States and the second at 1 site in Mexico. The protocol for both cohorts involved the same inclusion and exclusion criteria, hysteroscopic assessment, and observational end points. The study comprised a screening visit, diagnostic hysteroscopy, and follow-up phone call on day 7 to assess safety.

The study was conducted in accordance with Good Clinical Practice guidelines, as contained in the International Conference on Harmonization and US Code of Federal Regulations, and in accordance with the Declaration of Helsinki. The study protocol was approved by both central and local institutional review boards (IRBs) (Copernicus Group, Western IRB, Mercy Hospital IRB, Baylor Scott \& White Research Institute IRB, and the Research Committee of the Universidad Autónoma de Nuevo León). Written informed consent was obtained from all subjects for study participation, which included consent for still and video imaging of the hysteroscopic examination.

Women who were eligible for enrollment had completed their 36-month follow-up in the AEGEA Pivotal Clinical Trial (NCT01979861). Fig. 1 presents the subject disposition flow diagram. The water vapor ablation procedures were conducted between May 2014 and May 2015. The subjects were also required to have had normal Pap smear test results within the past 5 years.

Subjects were excluded from participation if they had undergone a repeat endometrial ablation, insertion of an

\section{Fig. 1}

Subject disposition. $\mathrm{PACE}=$ Post-Ablation Cavity Evaluation.

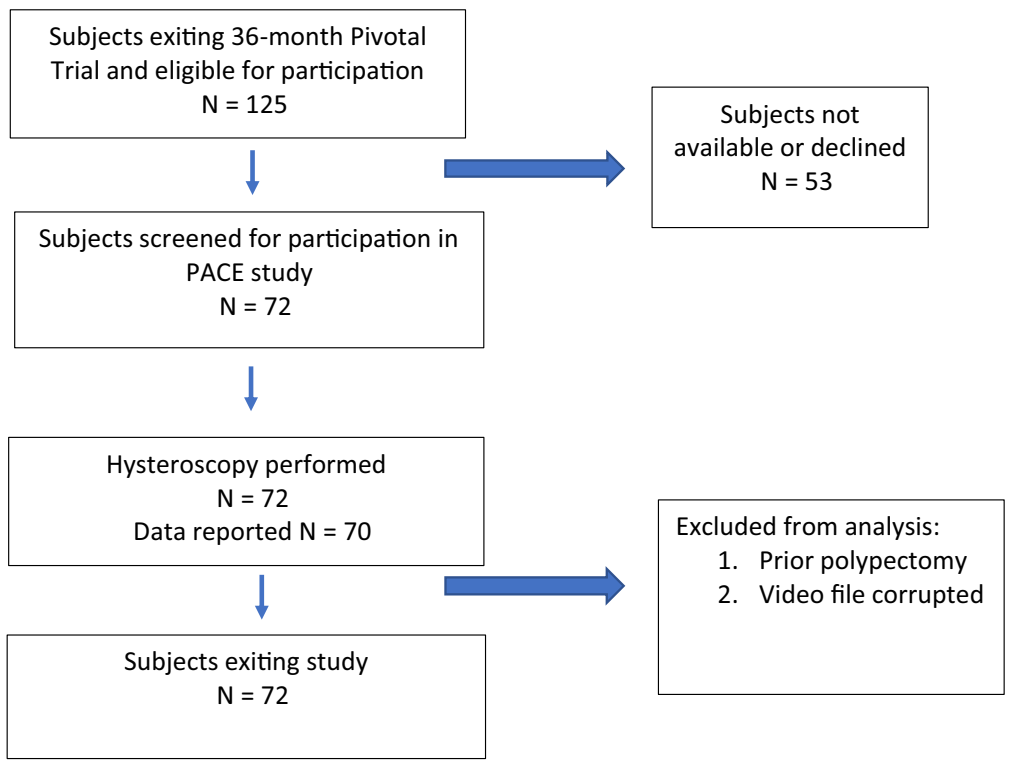


intrauterine device (IUD), or any other gynecologic procedure involving the application of an energy source or disturbance of endometrial tissue. Exclusion criteria also included pregnancy, as determined by a urine pregnancy test; evidence of an active sexually transmitted infection; active genitourinary tract infection; or suspected or confirmed gynecologic malignancy within the last 5 years. Of the 72 subjects who consented to study participation, 2 were excluded from the analysis: one because of a protocol violation as polypectomy and adhesiolysis had been performed before enrollment in the PACE protocol and the other because of a corrupt hysteroscopy video file, resulting in missing data for independent review. All enrolled subjects completed the 1-week follow-up.

The primary observational end point was the ability to access the uterine cavity and perform a diagnostic hysteroscopic examination. Other observational end points included the following: the ability to visualize the uterine cornua and tubal ostia and the presence and characterization of adhesions within the endometrial cavity. Finally, on the basis of a review of the hysteroscopy videos, the independent reviewer made a subjective assessment of the feasibility of Pipelle endometrial biopsy and IUD placement. The subjects' menstrual status was also recorded. Data were expressed as mean $\pm \mathrm{SD}$.

The safety end points were an assessment of diagnostic hysteroscopy-related serious adverse events and the overall rate and severity of all reported gynecologic adverse events.

Each investigator was a gynecologist with experience in performing hysteroscopy and endometrial ablation. All hysteroscopies were conducted in an office or outpatient setting using commercially available equipment. The selection of the hysteroscope and distension medium was at the discretion of the investigator. Hysteroscopy videos and still images were acquired in a standardized manner per established guidelines, which included instructions for careful video recording, beginning with entrance into the external cervical ostium. This allowed complete video documentation of adhesions from the cervical ostia to the uterine cavity. Lysis of adhesions was not allowed per the protocol.

Digital copies of the hysteroscopic images and videos were transferred to a core laboratory where a quality-control check was conducted. To ensure consistency and objectivity in image interpretation across all study sites, an experienced independent clinician, "independent reviewer," blinded to pivotal trial and ablation procedure data, evaluated the submitted videos according to the observational end points. The independent reviewer characterized uterine cavity adhesions as minimal, moderate, or severe according to the adhesion criteria by March et al [5], as shown in Table 1. An adhesion was defined as adherence of the uterine walls to each other, creating a bridge or band spanning the uterine cavity. Biopsy specimens were not collected and IUDs were not placed as part of this observational clinical study, but the independent reviewer subjectively projected whether these interventions were feasible.

In this study, categorical data were summarized using frequency tables, with numbers and percentages of subjects. For continuous variables, descriptive statistics included the number of subjects (n), mean, $\mathrm{SD}$, median, minimum, and maximum.

In addition, selection bias analysis was performed by an independent biostatistician (P.S.) to assess the comparability of the subjects enrolled in this study with the remaining eligible subjects who had completed their 36-month followup in the pivotal water vapor ablation study. A total of 126 subjects had completed their 36-month follow-up in the AEGEA Pivotal Trial. The PACE study enrolled 72 of these subjects. To evaluate selection bias, the characteristics of the 72 subjects who consented to participate in the PACE study were analyzed and compared with those of the remaining 54 subjects who had completed the pivotal trial. The following variables were assessed: demographics, site of service and analgesia used for the original procedure, and pain after the ablation procedure. Comparative analyses also included the following pivotal trial assessments at baseline and 12, 24, and 36 months: bleeding; quality of life, as measured by the Menorrhagia Impact Questionnaire; and treatment satisfaction.

\section{Results}

\section{Subject Demographics and Gynecologic History}

The mean age of the subjects was $43 \pm 5$ years (range 3354). The mean body mass index of the subjects was $29.9 \pm$ $7.0 \mathrm{~kg} / \mathrm{m}^{2}$ (range 17-51). Regarding race, 96\% (67/70) chose white, and regarding ethnicity, 30\% (21/70) listed Hispanic or Latino. For this cohort of PACE subjects, the mean pivotal trial baseline Pictorial Blood Loss Assessment Chart (by Higham et al [6]) score was $323.9 \pm 146.2$, and at the 12-month follow-up, it was $24.7 \pm 38.2$. The menstrual status data from the 24- and 36-month follow-up in the

\section{Table 1}

\section{Adhesion criteria}

Category Criteria

Minimal Less than one-fourth of uterine cavity, and thin or filmy adhesions, and ostial areas and upper fundus minimally involved or clear Moderate One-fourth to three-fourths of uterine cavity, with no agglutination of walls, and ostial areas and upper fundus only partially occluded Severe $\quad$ More than three-fourths of uterine cavity, or agglutination of walls or thick bands, or ostial area and upper cavity occluded 


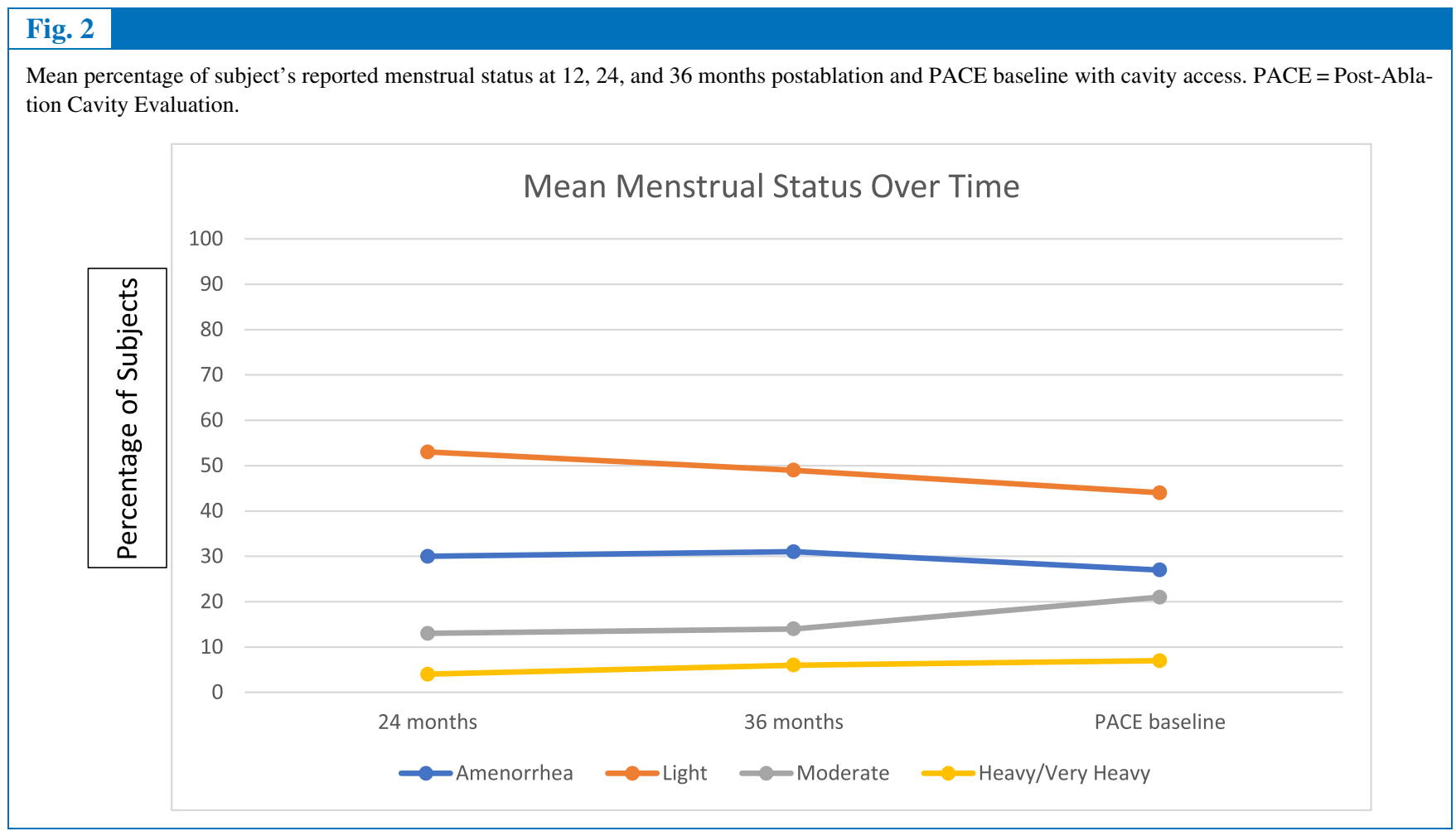

pivotal trial, as well as from PACE baseline, show consistency of the menstrual pattern across these time points (Fig. 2). At PACE baseline, 93\% (65/70) of subjects reported normal or no menstrual bleeding. The mean duration from the water vapor endometrial ablation procedure in the pivotal trial to the date of diagnostic hysteroscopy in the PACE study was $3.9 \pm 0.3$ (range 3.4-4.4) years.

\section{Diagnostic Hysteroscopy Procedure}

In most cases $(66 / 70,94 \%)$, diagnostic hysteroscopy was performed with a rigid hysteroscope. The hysteroscope's outer diameter ranged from 3 to $6 \mathrm{~mm}$. Normal saline $(0.9 \%)$ was used as the distension medium in all cases.

\section{Primary Observational End Point}

Uterine cavity access by hysteroscopy was achieved in $90 \%$ (63/70) of subjects. Access was limited to the endocervical canal in the remaining 7 subjects $(10 \%)$. The cornua and ostia were visualized in $79 \%(50 / 63)$ of cavities accessed or $71 \%$ (50/70) of all subjects, with $60 \%$ (30/50) bilateral and 40\% (20/50) unilateral visualization of the cornua and ostium. Representative photographs of uterine cavities with visualization of both the cornua and the ostia, with access but no visualization of the cornua, and with only endocervical access are shown in Fig. 3.

Adhesions were absent in 75\% (47/63) of uterine cavities that were accessed. Adhesions were characterized as "minimal" in $11 \%$ (7/63), "moderate" in 11\% (7/63), and "severe" in $3 \%$ (2/63) of subjects. No data regarding the severity of intrauterine adhesions could be obtained from the 7 subjects in whom cavity access was not possible. The independent reviewer subjectively determined that endometrial biopsy would be feasible in $89 \%$ (62/70) of subjects and that successful IUD placement would be feasible in $60 \%$ (42/70) of subjects. In one of the 2 subjects who had severe adhesions, the cavity was considered accessible, but the reviewer reprted that representative biopsy would not be feasible due to the adhesions.

\section{Menstrual Status}

Analysis of the potential correlation between cavity access and menstrual status was carried out on the basis of subject-reported menstrual status (none, light, moderate, heavy, or very heavy bleeding) at the time of study screening. Menstrual bleeding was further stratified by cavity access, with or without visualization of 1 or both cornua. As shown in Table 2 and Fig. 4, cavity access was achieved in $89 \%$ (17/19) of subjects with amenorrhea, 87\% (27/31) of subjects with light bleeding, 100\% (15/15) of subjects with moderate bleeding, and $80 \%$ (4/5) of subjects with heavy or very heavy bleeding. Spearman's rank correlation coefficient was 0.19 (95\% confidence interval -0.05 to 0.4 ), indicating no correlation between menstrual bleeding status and hysteroscopic access to the uterine cavity.

\section{Cavity Access and Menstrual Status in Subpopulations}

Of the 70 subjects evaluated, 29 (41\%) had large uterine cavities (10-12-cm uterine cavity lengths), FIGO type II to VI non-cavity-obstructing myomas $\leq 4 \mathrm{~cm}$ in diameter, 


\section{Fig. 3}

Long-term uterine cavity access. (a and b) Visualization of both cornua and ostia in the same subject; (c) Cavity access, no visualization of cornua; and (d) Endocervical access only. Right and left cornua (arrows).
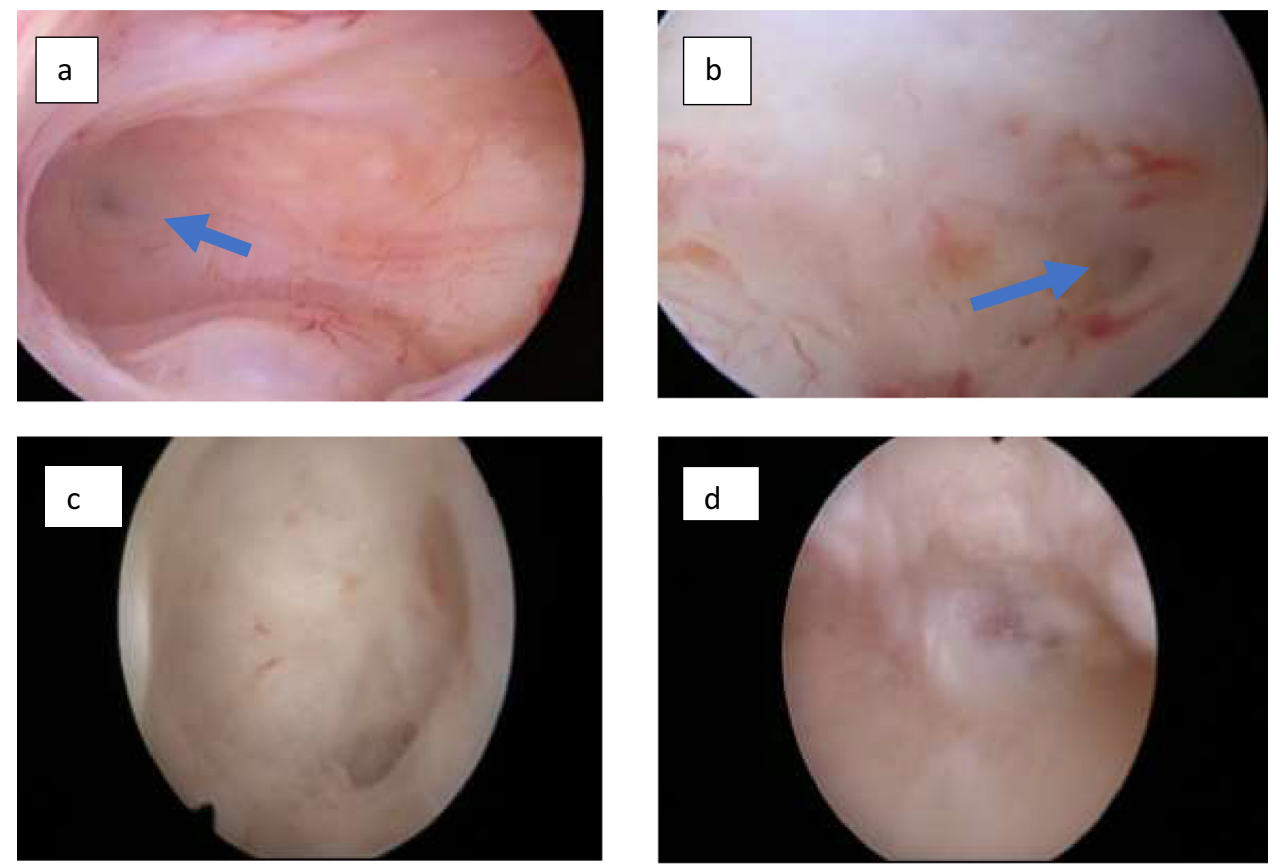

\section{Table 2}

Menstrual bleeding status and long-term cavity access

\begin{tabular}{lllll} 
Bleeding status & $\begin{array}{l}\text { Amenorrhea } \\
(\mathrm{n}=19)\end{array}$ & $\begin{array}{l}\text { Light } \\
(\mathrm{n}=31)\end{array}$ & $\begin{array}{l}\text { Moderate } \\
(\mathrm{n}=15)\end{array}$ & $\begin{array}{l}\text { Heavy/very heavy } \\
(\mathrm{n}=5)\end{array}$ \\
Uterine cavity access & $17(89)$ & $27(87)$ & $15(100)$ & $4(80)$ \\
\hline
\end{tabular}

Values are presented as $\mathrm{n}(\%)$.

and/or intratubal contraceptive inserts. Twelve subjects had large cavities, 11 had myomas, 3 had intratubal contraceptive inserts, and 3 had both large cavities and myomas. These subpopulations are traditionally not indicated for endometrial ablation treatment. Cavity access was achieved in $90 \%(26 / 29)$ of these subjects. Visualization of the cornua and ostia in the accessed cavities was $88 \%$ (23/26). In addition, at the time of baseline screening for the PACE study, 93\% (27/29) of these subjects reported normal (moderate), light, or no menstrual bleeding.

\section{Safety Results}

No hysteroscopy-related serious adverse events were reported. Of 72 subjects, 6 (8\%) developed 1 or more gynecologic adverse events. Only 1 gynecologic adverse event (dysmenorrhea) was reported as "severe"; this resolved in 2 days with no intervention. The remaining adverse events were pain, nausea, uterine cramping, and/or vaginal spotting.

\section{Analysis of Selection Bias}

No clinically relevant differences were detected between subjects enrolled in this study and eligible subjects who completed the pivotal trial. Bleeding assessments of the subjects in the 2 populations were nearly equivalent, with 94\% (68/72) reporting normal bleeding or less in the PACE cohort and 94\% (51/54) in the non-PACE cohort at 36month follow-up assessment of the pivotal study. The entire comparative cohort analysis is presented in Table 3.

\section{Discussion}

Recurrent heavy bleeding, cyclic pain, and postmenopausal bleeding may be associated with residual or regenerated endometrium, endometrial hyperplasia, endometrial cancer, and/or adhesions that commonly present after undergoing endometrial ablation. In separate prospective, longitudinal, clinical trials, Taskin et al [7] and Onoglu et al 


\section{Fig. 4}

Bleeding status and long-term cavity access.

\section{Bleeding Status and Percentage Cavity Access}

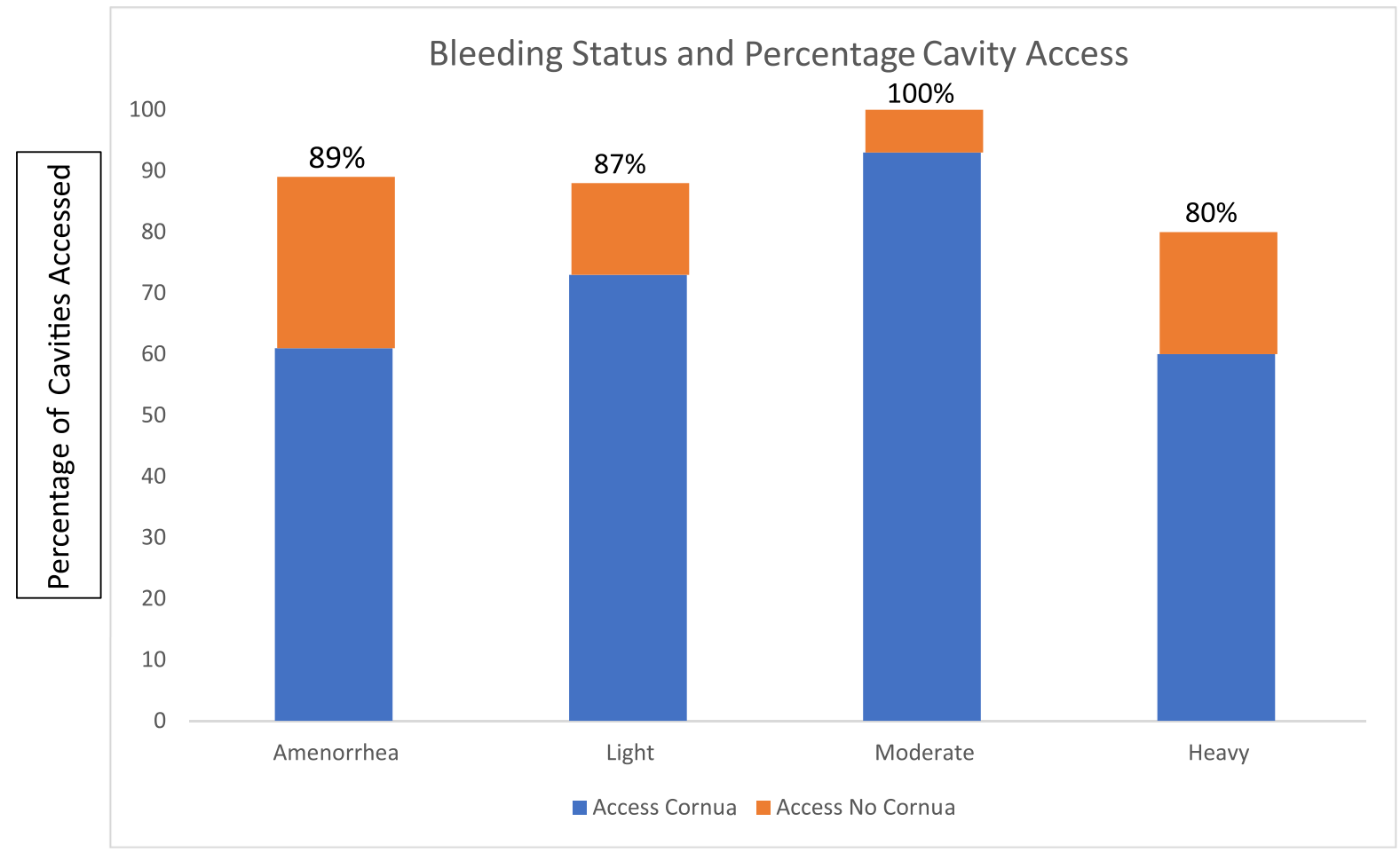

\begin{tabular}{|lll|}
\hline Table 3 & & \\
\hline Selection bias analysis & & \\
& & \\
Variable comparisons & PACE & Non-PACE \\
& $(\mathrm{N}=72)$ & $(\mathrm{N}=54)$ \\
& & \\
Mean age at pivotal baseline (yrs) & 39.5 & 40.6 \\
Race, $\mathrm{n}(\%)$ & & \\
White & $69(96)$ & $51(94)$ \\
Black & $2(3)$ & $2(4)$ \\
Asian & $1(1)$ & $1(2)$ \\
Mean BMI at pivotal baseline (kg/m $\left.{ }^{2}\right)$ & 29.5 & 29.3 \\
Mean gravidity at pivotal baseline & 3.3 & 3.1 \\
Mean parity at pivotal baseline & 2.8 & 2.6 \\
Mean uterine length at pivotal baseline (cm) & 9.1 & 8.9 \\
Dysmenorrhea at pivotal baseline, $\mathrm{n}(\%)$ & $56(78)$ & $49(91)$ \\
Mean PBLAC at pivotal baseline & 320.7 & 300.0 \\
Mean PBLAC at pivotal 12-month & 25.2 & 39.6 \\
Menstrual status of normal bleeding & $68(94)$ & $51(94)$ \\
or less at pivotal 36-month, $\mathrm{n}(\%)$ & & \\
Mean MIQ at pivotal baseline & 14.8 & 14.5 \\
Mean MIQ at pivotal 36-month & 6.2 & 6.4 \\
Patient satisfaction at pivotal 36-month, n (\%) & $65(90)$ & $50(93)$ \\
\hline BMI= body mass index; MIQ = Menorrhagia & Impact & Questionnaire; \\
PACE = Post-Ablation Cavity Evaluation; PBLAC=Pictorial & Blood Loss \\
Assessment Chart. & & \\
& & \\
\hline
\end{tabular}

[8] performed hysteroscopy in a total of 71 women at least 30 months after endometrial ablation with either hysteroscopic resection or rollerball ablation. A prominent finding at the time of hysteroscopy in both studies was focal endometrial regeneration. They concluded that such regeneration is "an expected development, not a failure of ablation." Other findings included dense intrauterine adhesions, total obliteration of the cavity, and atrophic endometrium.

Similarly, Turnbull et al [9] were able to identify residual endometrium using magnetic resonance imaging in $94.9 \%$ of 59 subjects who had undergone endometrial resection a mean of 34 months earlier. Of these, 22 subjects (37\%) were amenorrheic. These studies suggest that there is a need to access the uterine cavity to evaluate the residual or regenerated endometrium for diagnostic purposes after endometrial ablation.

Other issues related to intracavity scarring following endometrial ablation include cyclic pelvic pain from partial or complete menstrual obstruction and hematometra, which may occur months or years after the procedure [1]. Three large retrospective studies by Shavell et al [10], Longinatti et al [11], and Vilos et al [12] examined hysterectomy specimens from women seeking additional care following endometrial ablation. The indications for hysterectomy were recurrent bleeding in $12 \%$ to $51 \%$, cyclic pain in $20 \%$ to $64 \%$, and a combination of pain and bleeding in $23 \%$ to 
$38 \%$. These occurrences have recently been termed lateonset endometrial ablation failure. These manifestations, characterized by either recurrent bleeding or cyclic pain, have stimulated hysteroscopic attempts to recreate a cavity space for diagnosis or therapy, thus avoiding hysterectomy in patients desiring a less invasive option [13].

A 24-month feasibility trial by Thurkow et al produced promising results in terms of reduction of menstrual bleeding and continued satisfaction in 22 subjects who had undergone water vapor ablation [14]. Eleven of these subjects subsequently consented to hysteroscopic evaluation of the endometrial cavity as part of the PACE pilot study a mean of 4.1 years after the original procedure. In this pilot study, hysteroscopic access to the cavity and diagnostic assessment was possible in 82\% (9/11) of subjects [15].

A few studies have been conducted to evaluate access to the endometrial cavity after ablation. The methods of these studies vary widely, making it difficult to draw direct and meaningful comparisons with the findings from this study. Leung et al [16] and Luo et al [17], however, collected data on the menstrual status of subjects at the time of hysteroscopy after endometrial ablation using other modalities. They concluded that a greater degree of fibrosis and adhesion formation was correlated with a better reduction in menses after endometrial ablation. This is in contrast to the findings of our study using water vapor ablation. The subjects with amenorrhea had a high rate of uterine cavity access with a low incidence of adhesion formation. There was no correlation between bleeding status and uterine cavity access/adhesions.

The results from the PACE study further demonstrate that long-term access to the uterine cavity can be achieved in women who had undergone water vapor endometrial ablation 4 years prior. The majority of these women did not have intrauterine adhesions, which permitted uterine cavity access with visualization of the cornua and ostia. In most women, Pipelle biopsy and placement of an IUD were projected to be feasible. Moreover, menstrual bleeding status was not correlated with uterine cavity access and the ability to visualize the uterine cornua and ostia. Long-term effectiveness and cavity access were consistent in subjects with longer cavities (up to $12 \mathrm{~cm}$ ), uterine myomas (FIGO type II-VI up to $4 \mathrm{~cm}$ ), and/or intratubal contraceptive inserts, each of which has been traditionally excluded from endometrial ablation treatment.

Results from the pivotal trial [3] revealed that water vapor endometrial ablation is safe and provides effective long-term (over 3 years) bleeding reduction. Moreover, the results of this study, in combination with the PACE pilot study [15], show that water vapor ablation does not commonly compromise a clinician's ability to access the uterine cavity hysteroscopically for diagnosis or treatment.

The strengths of this study include its prospective nature. Regardless of the outcome of their ablation procedure, all pivotal subjects at each participating site were invited to participate in the current study, eliminating any bias based on the results of the original ablation. In addition, an accepted peer-reviewed scale was used to characterize uterine cavity adhesions. Analysis by a single experienced independent reviewer added objectivity and uniformity to the analysis.

Limitations of the study include its single-arm, observational design. In addition, the feasibility of a blind biopsy or IUD placement is strictly a projection made by the independent reviewer. Furthermore, although the inclusion of Pipelle endometrial biopsy introduces a first-line endometrial sampling technique familiar to practitioners and accepted by patients in an office setting, the limitations of this sampling method, especially in patients who have undergone prior endometrial ablation, are acknowledged [18]. Data on the need for anesthesia, type of analgesia used, and patient comfort during the hysteroscopic procedure were not collected in this study. Finally, follicle-stimulating hormone levels were not measured in the study subjects at baseline, and it is possible that undiagnosed menopause with onset after the ablation procedure could confound findings on whether a subject was amenorrheic because of the effect of the ablation treatment or because of menopause. However, the authors prospectively followed the bleeding status and noted the stability of reported menstrual effect from 12 months to 4 years after procedure in most subjects (Fig. 2).

In conclusion, it is clear that long-term access to the uterine cavity is an important consideration in the ongoing care of women who choose endometrial ablation for the treatment of heavy menstrual bleeding. Future research in the form of prospective, randomized, comparative trials would be useful to understand long-term outcomes among various endometrial ablation modalities. The ultimate goal should be the opportunity to offer ongoing minimally invasive diagnosis and treatment options to women who choose endometrial ablation.

\section{Acknowledgments}

The authors would like to thank the AEGEA PACE Investigator Group: Jeffrey Baker, MD (Clinical Research Prime, Idaho Falls, ID); Cindy Basinski, MD (CMB Research, Newburgh, IN); David Levine, MD (Mercy Hospital, St. Louis, MO); Andrea Lukes, MD (Carolina Women's Research and Wellness, Durham, NC); Charles Miller, MD (The Advanced Gynecologic Surgery Institute, Naperville, IL); and Kirk Brody, MD (Chattanooga Medical Research LLC, Chattanooga, TN); Peter Shabe, Sttatistician (Santa Clara, CA).

\section{References}

1. McCausland AM, McCausland VM. Long-term complications of endometrial ablation: cause, diagnosis, treatment, and prevention. $J$ Minim Invasive Gynecol. 2007;14:399-406.

2. Levie MD, Chudnoff SG. A prospective, multicenter, pivotal trial to evaluate the safety and effectiveness of the AEGEA vapor endometrial ablation system. J Minim Invasive Gynecol. 2019;26:679-687.

3. Hahn DW, Özisik MN. Heat Conduction. 3rd ed. Hoboken, NJ: John Wiley \& Sons Inc.; 2012 
4. Garza-Leal J, Hernandez I, Castillo L, Coad JE. AEGEA vapor-based endometrial ablation: perihysterectomy proof of concept. J Minim Invasive Gynecol. 2009;16:S65.

5. March CM, Israel R, March AD. Hysteroscopic management of intrauterine adhesions. Am J Obstet Gynecol. 1978;130:653-657.

6. Higham JM, O'Brien PM, Shaw RW. Assessment of menstrual blood loss using a pictorial chart. Br J Obstet Gynaecol. 1990;97:734-739.

7. Taskin O, Onoglu A, Inal M, et al. Long-term histopathologic and morphologic changes after thermal endometrial ablation. J Am Assoc Gynecol Laparosc. 2002;9:186-190.

8. Onoglu A, Taskin O, Inal M, et al. Comparison of the long-term histopathologic and morphologic changes after endometrial rollerball ablation and resection: a prospective randomized trial. J Minim Invasive Gynecol. 2007;14:39-42.

9. Turnbull LW, Jumaa A, Bowsley SJ, Dhawan S, Horsman A, Killick SR. Magnetic resonance imaging of the uterus after endometrial resection. Br J Obstet Gynaecol. 1997;104:934-938.

10. Shavell VI, Diamond MP, Senter JP, Kruger ML, Johns DA. Hysterectomy subsequent to endometrial ablation. J Minim Invasive Gynecol. 2012;19:459-464.

11. Longinotti MK, Jacobson GF, Hung YY, Learman LA. Probability of hysterectomy after endometrial ablation. Obstet Gynecol. 2008;112: $1214-1220$.
12. Vilos GA, Abu-Rafea B, Ettler HC, Ahmad R. Indications for hysterectomy and uterine histopathology following hysteroscopic endometrial ablation. J Minim Invasive Gynecol. 2005;12(Suppl):9.

13. Wortman M, Cholkeri A, McCausland AM, McCausland VM. Lateonset endometrial ablation failure-etiology, treatment, and prevention. J Minim Invasive Gynecol. 2015;22:323-331.

14. Thurkow A, Van Baal M, Van Eijndhoven M. Final results of a multicenter trial of safety and effectiveness of endometrial ablation with the AEGEA vapor system for the treatment of menorrhagia. J Minim Invasive Gynecol. 2015;22:S46.

15. van Eijndhoven H, Lenglet J, van Baal M, Thurkow A, Harris M. Post vapor ablation cavity evaluation: a pilot study. J Minim Invasive Gynecol. 2017;24:S85-S86.

16. Leung PL, Tam WH, Yuen PM. Hysteroscopic appearance of the endometrial cavity following thermal balloon endometrial ablation. Fertil Steril. 2003;79:1226-1228.

17. Luo X, Lim CE, Li L, Wong WS. Hysteroscopic appearance of endometrial cavity after microwave endometrial ablation. J Minim Invasive Gynecol. 2010;17:30-36.

18. Xie B, Qian C, Yang B, et al. Risk factors for unsuccessful officebased endometrial biopsy: a comparative study of office-based endometrial biopsy (Pipelle) and diagnostic dilation and curettage. J Minim Invasive Gynecol. 2018;25:724-729. 